University of Nebraska - Lincoln

DigitalCommons@University of Nebraska - Lincoln

Stephen Ducharme Publications

Research Papers in Physics and Astronomy

September 2003

\title{
Kinetics of ferroelectric switching in ultrathin films
}

G. Vizdrik

Institute of Crystallography, Russian Academy of Sciences, Moscow, Russia

Stephen Ducharme

University of Nebraska, sducharme1@unl.edu

V.M. Fridkin

University of Nebraska - Lincoln

S.G. Yudin

Institute of Crystallography, Russian Academy of Sciences, Moscow, Russia

Follow this and additional works at: https://digitalcommons.unl.edu/physicsducharme

Part of the Physics Commons

Vizdrik, G.; Ducharme, Stephen; Fridkin, V.M.; and Yudin, S.G., "Kinetics of ferroelectric switching in ultrathin films" (2003). Stephen Ducharme Publications. 7.

https://digitalcommons.unl.edu/physicsducharme/7

This Article is brought to you for free and open access by the Research Papers in Physics and Astronomy at DigitalCommons@University of Nebraska - Lincoln. It has been accepted for inclusion in Stephen Ducharme Publications by an authorized administrator of DigitalCommons@University of Nebraska - Lincoln. 


\title{
Kinetics of ferroelectric switching in ultrathin films
}

\author{
G. Vizdrik, ${ }^{1}$ S. Ducharme ${ }^{2}$ V. M. Fridkin, ${ }^{1,2}$ and S. G. Yudin ${ }^{1}$ \\ ${ }^{1}$ Institute of Crystallography, Russian Academy of Sciences, Moscow 117333, Russia \\ ${ }^{2}$ Department of Physics and Astronomy and Center for Materials Research and Analysis, University of Nebraska, \\ Lincoln, Nebraska 68588-0111, USA
}

(Received 5 September 2002; revised manuscript received 19 June 2003; published 23 September 2003)

\begin{abstract}
The kinetics of polarization switching in ultrathin ferroelectric polymer films exhibit a critical behavior; there is a pronounced slowing just above the coercive field and just below the critical temperature. The critical slowing is observed in the switching kinetics of ferroelectric Langmuir-Blodgett films of $70 \%$ vinylidenefluoride and $30 \%$ trifluoroethylene copolymer with thickness up to $30 \mathrm{ML}$. Thicker films exhibit an exponential dependence on the field and temperature that is normally associated with extrinsic switching by domain nucleation and growth. We show that the critical behavior arises naturally from the dynamics of homogeneous intrinsic switching in the context of mean-field theory. These results have important implications for the modeling and control of the ferroelectric films in nonvolatile computer memories and solid-state data storage media.
\end{abstract}

DOI: 10.1103/PhysRevB.68.094113

PACS number(s): 77.80.Fm, 77.80.Dj, 77.80.Bh, 77.22.Ej

A ferroelectric crystal maintains a permanent electric polarization that can be repeatedly switched between two stable states by an external electric field, thus exhibiting a polarization-electric field hysteresis loop analogous to the magnetization-magnetic field hysteresis loop exhibited by ferromagnetic crystals. The hysteresis loops are characterized by the magnitude of the remanent polarization achieved after saturation with a large electric field and by the magnitude of the coercive field, the minimum value of the electric field necessary to reverse, or switch, the polarization state. From the early studies of the kinetics of switching in ferroelectric crystals, ${ }^{1,2}$ it has been apparent that switching is almost invariably an extrinsic process involving the inhomogeneous nucleation of small domains of reversed polarization, usually initiated at crystal boundaries or defects, and subsequent growth of these domains to fill the crystal. Nucleation-limited extrinsic switching is characterized by an exponential increase in the switching rate with increased temperature and electric field, ${ }^{1}$ characteristic of an activated process, ${ }^{3}$ and is usually achieved with external fields in the range 0.1 to $50 \mathrm{MV} / \mathrm{m}^{4}$ Strictly speaking, extrinsic switching does not have a true threshold coercive field because the activation of nucleation permits switching at arbitrarily small fields, given enough time, but switching experiments are typically carried out with an ac field and so the apparent coercive field is actually a function of frequency. ${ }^{5}$

In the absence of nucleation, switching an ideal ferroelectric crystal with uniform polarization requires the application of an enormous coercive field. We call this the intrinsic switching mechanism, and the associated threshold field the intrinsic coercive field. ${ }^{4,6}$ The expected value of the intrinsic coercive field is of order $100 \mathrm{MV} / \mathrm{m}$ in most ferroelectrics. ${ }^{4}$ Intrinsic switching does not occur below the intrinsic coercive field because the constituent crystal dipoles are highly correlated-they tend to switch coherently or not at all. Only recently has a ferroelectric system been found to exhibit the characteristics of intrinsic switching: ultrathin LangmuirBlodgett films of a vinylidene fluoride-trifluoroethylene copolymer. ${ }^{6}$ Extrinsic switching is apparently suppressed in the ultrathin films because nucleation is inhibited as the reduced film thickness limits nucleation volume. The measured coercive field in these films reaches $1 \mathrm{GV} / \mathrm{m},{ }^{6}$ much larger than the values of $50 \mathrm{MV} / \mathrm{m}$ typically found in thicker solvent-formed films of the same polymers, ${ }^{7}$ and in good agreement with the predictions of the mean-field theory of ferroelectrics. ${ }^{8,9}$ Notably, the intrinsic switching process takes seconds, ${ }^{6}$ as opposed to microseconds typically achieved for extrinsic switching observed at much lower fields in thicker films ${ }^{10}$ or in specially prepared LangmuirBlodgett (LB) films, ${ }^{11}$ of the same materials.

Here we report the results of a study of the switching kinetics in the ultrathin copolymer LB films, revealing a critical slowing as the applied field approaches the coercive field from above or as the temperature approaches the critical temperature from below. This is qualitatively different from the kinetics of extrinsic switching, which lack a true coercive field and speed up with increased temperature or increased field. We show how the observed critical behavior arises naturally from the mean-field theory of ferroelectricity, and dispel the misconception that the intrinsic switching time should be comparable to the ferroelectric relaxation time. ${ }^{12,13}$

In order to explain intrinsic switching dynamics, we first describe the intrinsic polarization-field $P(E)$ hysteresis loop of the ferroelectric crystal. The crystal state at constant electric field $E$ and temperature $T$ and at zero stress can be described by a Gibbs free energy $G(P)=a\left(T-T_{0}\right) P^{2} / 2$ $+\beta P^{4} / 4+\gamma P^{6} / 6-E P$. The coefficients for a ferroelectric with a first-order phase transition are $a>0, \beta<0$, and $\gamma$ $>0$, and are assumed independent of temperature near $T_{0}$. The zero-field equilibrium polarization states are $P$ $= \pm P_{\mathrm{S}}(T)$ below the equilibrium phase transition temperature $T_{\mathrm{C}}=T_{0}+3 \beta^{2} / 16 \gamma a$, but metastable polarization can be maintained up to the critical temperature $T_{1}=T_{0}+\beta^{2} / 4 \gamma a$. A steady-state $P(E)$ curve, obtained by minimizing the free energy $G(P)$, is shown in Fig. 1(a). The heavy lines (AB and $\mathbf{A}^{\prime} \mathbf{B}^{\prime}$ ) denote fully stable states, the thin lines ( $\mathbf{B C}$ and $\left.\mathbf{B}^{\prime} \mathbf{C}^{\prime}\right)$ denote metastable states, and the dotted line $\left(\mathbf{C O} \mathbf{C}^{\prime}\right)$ denotes dynamically unstable states. Consider starting with 


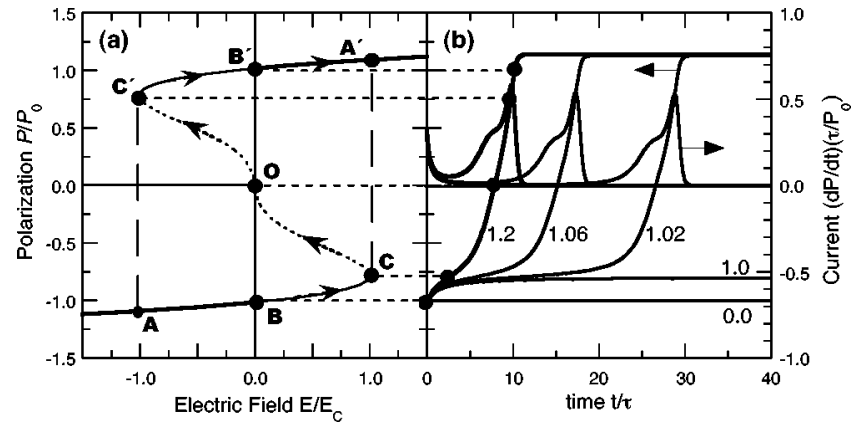

FIG. 1. (a) The polarization-field $P(E)$ curve calculated from Eq. (1) for $T \approx T_{0}$. The arrows show the path $P\left(E^{\prime}\right)$ taken during intrinsic switching, beginning at point $B$ and ending to the right of point $A^{\prime}$. The vertical dashed lines show the values $E / E_{\mathrm{C}}= \pm 1$ marking the limits of the quasi-steady-state hysteresis loop. (b) Time evolution of the normalized polarization $P / P_{0}$ and current $(\mathrm{d} P / \mathrm{d} t) /\left(\tau / P_{0}\right)$ during switching at $T=T_{0}$ for several values of the normalized electric field $E / E_{\mathrm{C}}$ given by the numbers next to each curve. The numbers give the value of the normalized switching field $E / E_{\mathrm{C}}$. The horizontal dashed lines connect related points in (a) and (b) as described in the text. The reference polarization is $P_{0}$ $=P_{\mathrm{S}}\left(T_{0}\right)=\operatorname{sqrt}(|\beta| / \gamma)$.

the sample in state $\mathbf{B}$ and applying an opposing electric field $E$. If $E<E_{\mathrm{C}}$, then the polarization will remain on the line $\mathbf{B C}$, returning to $\mathbf{B}$ after the field is removed. If $E>E_{\mathrm{C}}$, then the polarization will switch to the upper curve $\mathbf{A}^{\prime} \mathbf{B}^{\prime}$ as indicated by the vertical dashed line at $E / E_{\mathrm{C}}= \pm 1$, returning to $\mathbf{B}^{\prime}$ after the field is removed. The reverse process, going from $\mathbf{B}^{\prime}$ to $\mathbf{B}$, is essentially the same. If the crystal remains macroscopically homogenous (there are no independent domains) then the polarization will evolve along the line $\mathbf{C O C}^{\prime}$ in switching between alternate states. A quasistatic hysteresis loop is obtained by measuring the polarization while applying a low-frequency alternating voltage with an amplitude $E>E_{\mathrm{C}}$ so that the polarization will cycle repeatably in the sequence $\mathbf{A}-\mathbf{B}-\mathbf{C}-\mathbf{A}^{\prime}-\mathbf{B}^{\prime}-\mathbf{C}^{\prime}-\mathbf{A}$.

The intrinsic switching dynamics can be derived from the Lagrange equation for the Gibbs free energy $G(P)$, similar to the equation obtained earlier by Landau and Khalatnikov ${ }^{14}$ (LK) to describe anomalous sound absorption connected with a second-order phase transition. This equation, adapted for the first-order ferroelectric, ${ }^{15}$ is

$$
\xi \frac{d P}{d t}=-\frac{\partial G}{\partial P}=-a\left(T-T_{0}\right) P-\beta P^{3}-\gamma P^{5}+E,
$$

where $\xi$ is the polarization damping constant and should depend on temperature. Analyses of the Lagrangian dynamics of ferroelectric switching were reported in several recent papers, ${ }^{15-17}$ all of which concerned some variation of Eq. (1). Because Eq. (1) is derived from the thermodynamic free energy, it applies only in the quasi-static case when the system evolves slowly relative to microscopic fluctuations, i.e., when the system is over-damped. Therefore, like LK and the recent analyses, ${ }^{15-18}$ we have assumed that the inertial term, which is proportional to the second time derivative of the polarization, is negligible. Note that Eq. (1) applies only in the case of zero stress. Otherwise, one must solve it simul- taneously with a similar dynamical equation for the stress, with stress-polarization cross terms in both equations. ${ }^{9}$

Now consider a switching event beginning with the sample initially polarized in the negative direction at zero applied field [point B in Fig. 1(a)] and then abruptly applying a positive field $E$. The evolution of the polarization $P(t)$ during switching is readily obtained by numerical integration of Eq. (1) and is shown in Fig. 1(b) along with the current transients $\mathrm{d} P(t) / \mathrm{d} t$ for several values of the normalized switching field $E / E_{\mathrm{C}}$. Note that there is switching only for $E / E_{\mathrm{C}}>1$. If we define a dynamical variable $E^{\prime}=E$ $-\xi \mathrm{d} P(t) / \mathrm{d} t$, then the state traces out a $P\left(E^{\prime}\right)$ curve, like the

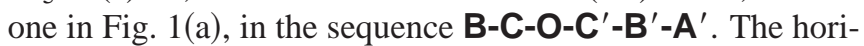
zontal dashed lines in Fig. 1 connect corresponding points on the $P\left(E^{\prime}\right)$ curve to the polarization transient for $E / E_{\mathrm{C}}$ $=1.2$. Notice that the polarization evolution is slowest (minimum current), when the state passes through point $\mathbf{C}$, and fastest, (maximum current) at $\mathbf{C}^{\prime}$. The slowing near point $C$ was noted by Ricinschi et al. ${ }^{16}$ from analysis of switching kinetics for a second-order ferroelectric and by Tan et al. ${ }^{15}$ for a first-order ferroelectric.

There are two convenient points for denoting the switching time, the time $t_{0}$ to reach zero polarization (B-O) or the time $t_{\mathrm{m}}$ to reach maximum current $\left(\mathbf{B}-\mathbf{C}^{\prime}\right)$. From the numerical analysis of Eq. (1) for a switching field $E$ just above $E_{\mathrm{C}}$ or for temperature $T$ just below $T_{1}$, we find that the reciprocal switching time $t_{0}$ has a square-root critical dependence of the form

$$
\frac{1}{t_{0}} \cong \frac{1}{\tau}\left(\frac{E}{E_{\mathrm{C}}}-1\right)^{1 / 2}\left(1-\frac{T-T_{0}}{T_{1}-T_{0}}\right)^{1 / 2}
$$

where $\tau \approx 6.3 \gamma \xi / \beta^{2}$. The switching time $t_{0}$ tends to infinity as $E \rightarrow E_{\mathrm{C}}$ from above or $T \rightarrow T_{1}$ from below. Well below $T_{1}$ or well above $E_{\mathrm{C}}$ the reciprocal switching time $t_{0}$ is linear in both $T$ and $E$. The square root and linear regimes are evident in the plots of the reciprocal switching time vs. field [Fig. $2(\mathrm{a})]$ and vs. temperature [Fig. 2(b)]. The critical slowing near $E_{\mathrm{C}}$ and $T_{1}$ was noted before, ${ }^{15,17}$ but without an indication of the functional form. We have recently learned of an analytical calculation showing that the square root behavior arises from the kinetics at the slowest point C. ${ }^{19}$ The switching time $t_{\mathrm{m}}$ is slightly longer than $t_{0}$, but has approximately the same dependence on temperature and field below $T_{\mathrm{C}}$. In the case of the first-order ferroelectric, the switching transients are complicated by the existence of double hysteresis loops, resulting in double current peaks just below $T_{\mathrm{C}}$. Between $T_{\mathrm{C}}$ and $T_{1}$ the zero-field equilibrium state is paraelectric $\left(P_{\mathrm{S}}=0\right)$, so the film can get stuck in this state for switching fields just above $E_{\mathrm{C}}$, but not large enough to repolarize the crystal, as was pointed out by Tan et al. ${ }^{15}$ Our analysis of Eq. (1) in the case of the second-order ferroelectric also shows a square-root critical behavior similar to Eq. (2).

The studies of intrinsic switching kinetics were made on ferroelectric LB films of a random copolymer consisting of $70 \%$ vinylidene fluoride and $30 \%$ trifluoroethylene P(VDFTrFE 70:30). ${ }^{20,21}$ Prior studies of the LB copolymer films 

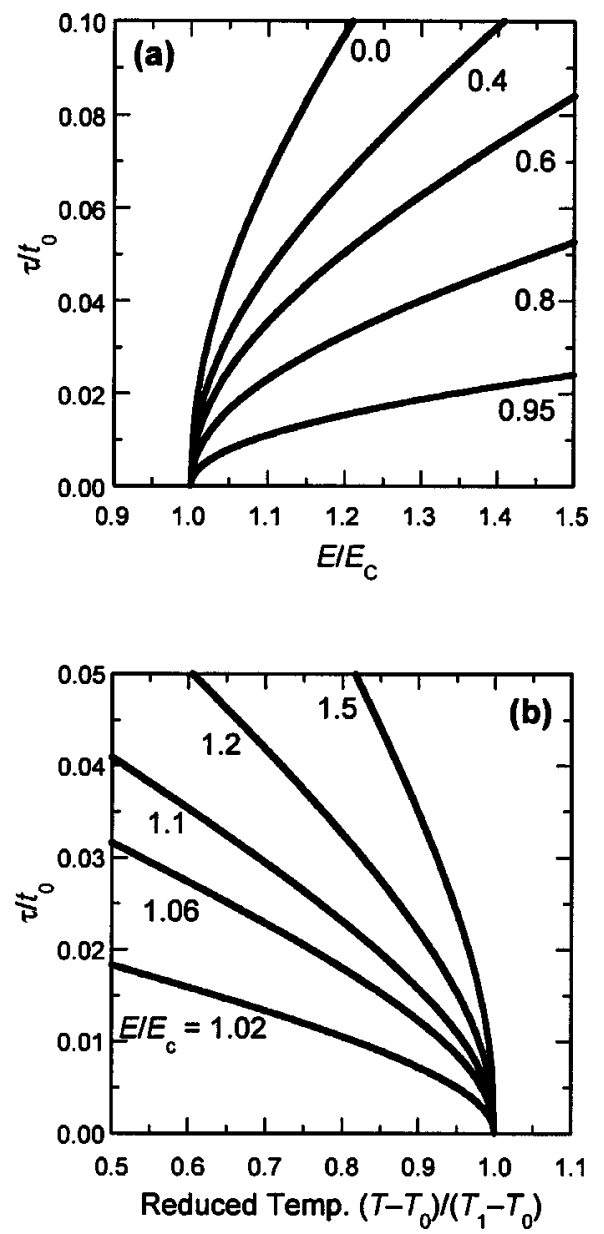

FIG. 2. (a) Dependence of the reciprocal switching time $\tau / t_{0}$ on the electric field $E / E_{\mathrm{C}}$ calculated from Eq. (1) at several values of the reduced temperature $\left(T-T_{0}\right) /\left(T_{1}-T_{0}\right)$ given by the numbers next to each curve. (b) Dependence of the reciprocal switching time $\tau / t_{0}$ on the reduced temperature $\left(T-T_{0}\right) /\left(T_{1}-T_{0}\right)$ calculated from Eq. 1 at several values of the reduced electric field $E / E_{\mathrm{C}}$ given by the numbers next to each curve.

confirmed the presence of polarization hysteresis, ${ }^{20}$ and a first-order paraelectric-ferroelectric phase transition at $T_{\mathrm{C}}$ $\approx 80^{\circ} \mathrm{C}$ even in films as thin as $1 \mathrm{~nm} .{ }^{22}$ The samples for the present study were made as follows. The $\mathrm{P}(\mathrm{VDF}-\mathrm{TrFE}$ 70:30) copolymer was dissolved to a concentration of $0.05 \%$ by weight in dimethylsulfoxide, dispersed on the surface of an ultrapure $(18 \mathrm{M} \Omega-\mathrm{cm})$ water subphase, and slowly compressed to a surface pressure of $5 \mathrm{mN} / \mathrm{m}$. The multilayer samples were formed by the horizontal (Schaefer) variation of Langmuir-Blodgett deposition on a glass substrate coated with 50 -nm-thick, 1-mm-wide stripes of evaporated aluminum. A second set of aluminum stripes was evaporated at right angles to the first set on top of the film, thus forming an array of independently addressable ferroelectric capacitors. The samples were then annealed at $120^{\circ} \mathrm{C}$ for $1 \mathrm{~h}$ and cooled to room temperature at a rate of $1{ }^{\circ} \mathrm{C}$ per h. Film fabrication methods are described in greater detail in Ref. 21. These films had coercive voltages $V_{\mathrm{C}}\left(=E_{\mathrm{C}}\right.$ times the film thickness), measured at $25^{\circ} \mathrm{C}$, of $6.6 \pm 0.1 \mathrm{~V}$ and $5.0 \pm 0.1 \mathrm{~V}$ for
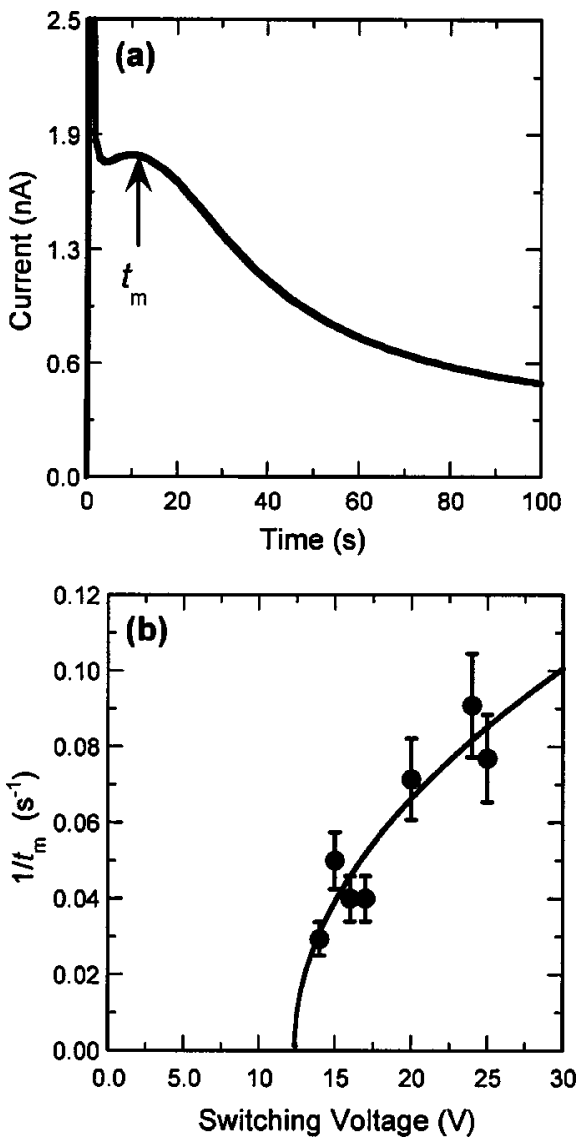

FIG. 3. (a) Switching current transient from 30-ML film 1 recorded with a $24-\mathrm{V}$ switching pulse at $25^{\circ} \mathrm{C}$. (b) Dependence of the reciprocal switching time $1 / t_{\mathrm{m}}$ on switching voltage, measured with $30 \mathrm{ML}$ film 1 at $25^{\circ} \mathrm{C}$. The solid line is a least-squares fit of the data to Eq. (2).

10-ML films 1 and 2, 10 $\pm 0.5 \mathrm{~V}$ for both 30-ML films 1 and 2 , and $25 \pm 0.5 \mathrm{~V}$ for a $100-\mathrm{ML}$ film. The thickness of these samples was not measured directly, but assuming a thickness of $0.5 \mathrm{~nm}$ per monolayer ${ }^{22}$ means that a $10-\mathrm{ML}$ film is $5 \mathrm{~nm}$ thick and so an applied voltage of $5 \mathrm{~V}$ would produce an internal field of $1 \mathrm{GV} / \mathrm{m}$. The temperature dependence of the coercive field for these films was consistent with prior studies of the intrinsic coercive field in similar samples. ${ }^{6}$

Two methods were used to measure the switching time. The first method consisted of measuring the current transients during switching and determining the time $t_{\mathrm{m}}$ of peak current. Figure 3(a) shows a representative switching current transient from 30-ML film 1. The critical behavior is evident in Fig. 3(b); the reciprocal switching time $1 / t_{\mathrm{m}}$ decreases precipitously toward zero as the field decreases towards the coercive voltage $V_{C}$. The solid line is a least-squares fit to Eq. (2), yielding the values for the time constant $\tau=11.9$ $\pm 0.5 \mathrm{~s}$, the damping constant ${ }^{23} \xi=3.6 \pm 1 \times 10^{10} \mathrm{Vms} / \mathrm{C}$, and the coercive voltage $V_{\mathrm{C}}=12.3 \pm 1 \mathrm{~V}$, the latter in good agreement with the quasistatic hysteresis loop measurement of $V_{\mathrm{C}}=10 \pm 0.5 \mathrm{~V}$.

The second method for measuring the switching time is to apply a short switching pulse of fixed duration and then recording the film pyroelectric response at zero bias using the 


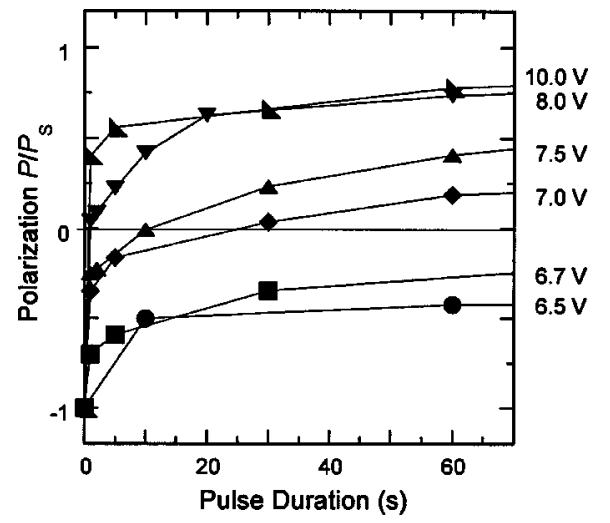

FIG. 4. Polarization measured by the pyroelectric method after switching as a function of pulse duration measured with a 10-ML film 1 at $25^{\circ} \mathrm{C}$ for different values of the applied voltage. The amplitudes of the voltage pulse for each curve were $6.5 \mathrm{~V}$ (circles), 6.7 V (squares), 7.0 V (diamonds), 7.5 V (triangles), 8.0 V (inverted triangles), and $10 \mathrm{~V}$ (wedges).

Chynoweth pyroelectric technique, ${ }^{24}$ which consists of periodically heating the sample with a helium-neon laser at a $632.8-\mathrm{nm}$ wavelength modulated at $1 \mathrm{kHz}$ by an optical chopper, and measuring the resulting short-circuit ac current from the sample with a lock-in amplifier. Strictly speaking, here we measure not the microscopic polarization $P$, but the pyroelectric response $\mathrm{d} P / \mathrm{d} T$, which is proportional to net film polarization. The basis and detailed technique of the pyroelectric measurement method were described in greater detail previously. ${ }^{24,25}$ The pulse switching method works well even for very slow switching (i.e., near $E_{\mathrm{C}}$ or $T_{1}$ ) when the switching transients are too small to measure directly in the presence of sample conductance and noise. Figure 4 shows the dependence of this switched polarization on the pulse amplitude and duration for 10 -ML film 1 at $25^{\circ} \mathrm{C}$. The switching time is taken as the pulse duration necessary to change the sign of the polarization, essentially the same as the above definition of $t_{0}$. The polarization curves in Fig. 4 show clearly the qualitative change in behavior near the coercive voltage. With an opposing voltage of $6.7 \mathrm{~V}$ or less, the sample polarization did not change sign, even after more than 5 min of field application, indicating that the voltage was at or just below the coercive voltage, in good agreement with the value $V_{\mathrm{C}}=6.6 \pm 0.1 \mathrm{~V}$ determined from the quasistatic hysteresis loops. ${ }^{26}$ The switching time $t_{0}$ in the 10-ML films increases markedly as either $V \rightarrow V_{\mathrm{C}}$ from above (Fig. 5 ) or as $T \rightarrow T_{1}$ from below (Fig. 6), as predicted by Eq. (2). The lower line in Fig. 5 is obtained by integrating Eq. (1) and adjusting the vertical scale to fit the data from the two 10-ML samples, yielding the values for the time constant $\tau$ $=12.6 \pm 0.5 \mathrm{~s}$ and the damping constant $^{23} \quad \xi \approx 3.8 \pm 1$ $\times 10^{10} \mathrm{~V} \mathrm{~ms} / \mathrm{C}$, in good agreement with the value obtained from the current transient measurements on $30 \mathrm{ML}$ film 1 (Fig. 3). Figure 6 shows that the reciprocal switching time in 10-ML film 1 decreases more sharply than expected [compare Fig. 2(b)] as $T$ increases, falling off nearer to $T_{\mathrm{C}}$ $\approx 80^{\circ} \mathrm{C}$, well below $T_{1} \approx 95^{\circ} \mathrm{C}$. The additional slowing near $T_{\mathrm{C}}$ may be due to additional damping from critical fluctua-

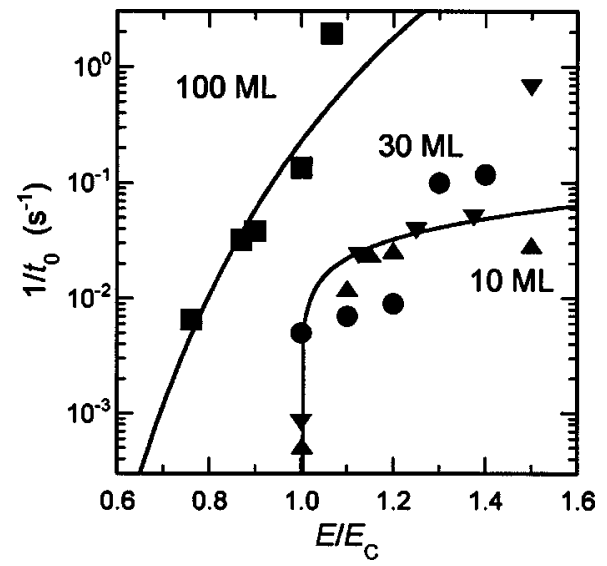

FIG. 5. Dependence of the reciprocal switching time $1 / t_{0}$ on the switching field $E / E_{\mathrm{C}}$, measured at $25^{\circ} \mathrm{C}$, with 10 -ML film 1 (up triangles), 10-ML film 2 (down triangles), $30 \mathrm{ML}$ film 2 (circles) and the $100 \mathrm{ML}$ film (squares). The lower line shows the functional form of the intrinsic switching fit to the data from the 10-ML films, and the upper line shows the form of extrinsic switching fit to the data from the 100-ML film.

tions connected with the phase transition and phase coexistence between $T_{\mathrm{C}}$ and $T_{1}$. Critical fluctuations are beyond the scope of the mean-field theory. This is a potentially fruitful regime for future experimental and theoretical study.

Prior studies suggest that extrinsic mechanisms dominate switching in LB films thicker ${ }^{6}$ than $30 \mathrm{ML}$ and in all the solvent-formed films, ${ }^{10}$ because measured coercive fields in these films are much lower, and switching is generally much faster. But, even some films thinner than $30 \mathrm{ML}$ do not show such clear critical dependence on the switching voltage, perhaps because nucleation is not fully suppressed. More telling is the dependence of the switching time on temperature for films with different thickness (Fig. 6). The 100-ML film exhibits clear extrinsic behavior, switching even at fields well below the nominal coercive field (see Fig. 5), and with the

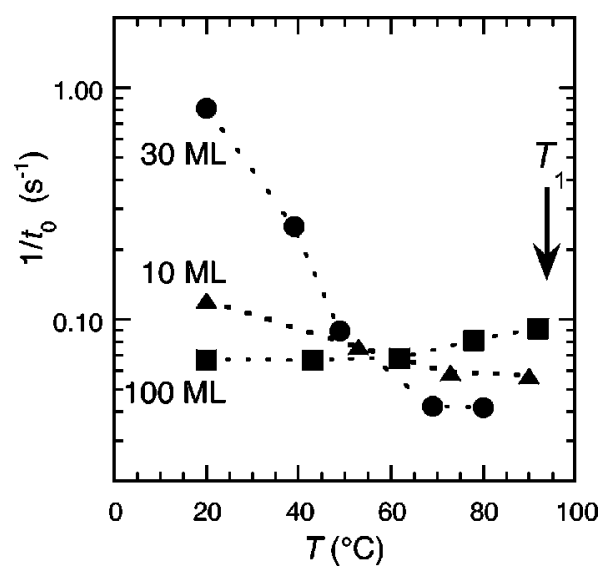

FIG. 6. Dependence of the reciprocal switching time $1 / t_{0}$ on temperature, measured at a constant value of the normalized switching electric field $E / E_{\mathrm{C}}$, from 10 -ML film 1 at $E / E_{\mathrm{C}}=1.06$ (triangles), 30-ML film 2 at $E / E_{\mathrm{C}}=1.1$ (circles), and the $100-\mathrm{ML}$ film at $E / E_{\mathrm{C}}=1.0$ (squares). 
usual exponential dependence of the reciprocal switching time on field and temperature $1 / t_{0} \alpha \exp [-(c / T+b) / E]$, where $c$ and $b$ are constants. While the switching time in 30-ML film 2 shows a mostly intrinsic critical dependence on field (Fig. 5), the reciprocal switching time also decreases, but not toward zero, as $T \rightarrow T_{1}$, indicating that switching proceeds by a combination of intrinsic and extrinsic mechanisms. The exponential form has been observed in studies of nucleationlimited ferroelectric switching from the early work of Merz, ${ }^{1}$ including the prior studies of solvent-formed films of PVDF and its copolymers. ${ }^{10,27}$ (In some cases, domain-wall motion is the limiting process in extrinsic switching, so that the reciprocal switching time increases linearly with field. ${ }^{1}$ ) At fields very close to $E_{\mathrm{C}}$, the exponential and critical forms are very distinct because the exponential form passes smoothly through $E_{\mathrm{C}}$ and the critical form drops abruptly toward zero. This is particularly evident in the data from the 10-ML films (triangles on Fig. 5).

At present we lack detailed information on the polarization distribution in the ferroelectric LB films within the individual crystallites, which are of order $100-500 \mathrm{~nm}$ in size. Therefore, we cannot exclude contributions to switching from domain nucleation and growth in films thinner than about $30 \mathrm{ML}$, nor can we confirm that these processes occur in thicker films exhibiting an activated behavior. Further, it is possible that, due to film inhomogeneity, the crystallites have a distribution of properties (i.e., the values of $\xi, a, \beta, \gamma$, and $T_{0}$ are inhomogeneous). The sharpness of the critical behavior in field suggests that the inhomogeneity in the coercive field is small, but it is possible that the damping constant $\xi$ is more sensitive to microsctructure. To help resolve these questions, we are currently developing an imaging technique with sufficient spatial and temporal resolution to image switching kinetics the crystallites.

There remains the important question of why switching in the thinnest LB films is so slow, over six orders of magnitude slower than in the solvent-formed films. There is no a priori reason that the intrinsic switching time is in any way related to the extrinsic switching time. Originally, LK restricted the mean-field dynamics represented by Eq. (1) to small perturbations from equilibrium, ${ }^{14}$ in which case the damping constant $\xi$ is proportional to the ultrasonic attenuation coefficient. The damping constant itself cannot be obtained from the mean field theory but must be calculated from a microscopic approach, like molecular dynamics. Later interpretations attributed this attenuation to damping of the soft phonon mode in ferroelectrics. ${ }^{28,29}$ Recent papers ${ }^{12,16,18}$ continue to claim that the highly nonequilibrium process of switching must also proceed in a time comparable to the quasiequilibrium LK relaxation time, which is of order $300 \mathrm{ps}$ in the ferroelectric copolymers. ${ }^{30}$ According to this interpretation, intrinsic switching should be much faster than extrinsic switching, and therefore the large observed coercive fields in the thinnest LB copolymer films are not connected with intrinsic switching, but with some pinning mechanism. ${ }^{12} \mathrm{We}$ argue $^{13}$ against this interpretation on the grounds that collective polarization reversal in intrinsic switching, where the physical dipoles must rotate by $180^{\circ}$, faces considerably larger damping due to impediments by neighboring chains not encountered in the small-perturbation case. The dipoles, being correlated according to the order parameter $P$, cannot switch collectively faster than the lattice can expand because of the large interchain steric hindrance. A more general model would consider microscopic polarization fluctuations, which produce large strains, considerably impeding reorganization of the polarization state. Then, the slower evolution of the macroscopic polarization is a result of microscopic fluctuations, which greatly dampen its evolution. (In the case of a single chain, the boundary between different polarization states amounts to a kink or soliton that can travel at quite high speed, ${ }^{31}$ but interchain van der Waals and electrostatic interactions greatly impede kink formation and motion. ${ }^{32}$ ) A fluctuation damping model might be suitable for displacive ferroelectrics like barium titanate as well. The same considerations should apply to the dynamics of the phase transition, where large molecular rotations (large strains) are necessary to convert between the all-trans ferroelectric phase and the trans-gauche paraelectric phase. Detailed measurements of phase transition kinetics in P(VDFTRFE) copolymers by Toda et $a l_{.}^{33}$ show that the time to bring about such a reorganization is of order 10-100 seconds, comparable to the intrinsic switching time near $T_{\mathrm{C}}$. Therefore, we propose that the Lagrange dynamics of Eq. (1) can be applied to intrinsic switching, but the relevant damping constant $\xi$ is large and so the characteristic switching time $\tau$ is much larger than the LK relaxation time. Calculation of the damping constant $\xi$ would require a thorough accounting for the molecular dynamics, considering both intrachain and interchain interactions, for large deviations from crystal equilibrium, and is beyond the scope of this paper.

Summing up the results, we have observed a critical dependence of the ferroelectric switching kinetics on field and temperature in ultrathin Langmuir-Blodgett films of P(VDFTrFE 70:30) of about $30 \mathrm{ML}$ or thinner, and shown how these kinetics arise from the dynamics of mean-field theory of ferroelectrics in the case of homogeneous intrinsic switching. The intrinsic and extrinsic mechanisms are readily distinguished because of the dramatically different dependence on field and temperature. From these results, we come to the conclusion that the kinetics of intrinsic polarization reversal are well described by the Lagrangian dynamics of intrinsic switching within the mean-field formalism. The reason for the apparent crossover from intrinsic to extrinsic switching in the LB films at about $30 \mathrm{ML}$ thickness ${ }^{6}$ is not clear-some films thinner than $30 \mathrm{ML}$ do not show a critical behaviorbut scaling studies of the extrinsic coercive field above the $30 \mathrm{ML}^{34}$ suggested that the suppression of nucleation in thinner films raises the extrinsic coercive field until, at about 30 ML and thinner, it exceeds the intrinsic coercive field, so intrinsic switching dominates. ${ }^{6}$ The present results have major implications for data storage devices based on ultrathin ferroelectric films. As manufacturers reduce film thickness and feature size in a quest for higher density, there may be a similar transition toward slower intrinsic switching like the millionfold slowing observed in the ultrathin ferroelectric polymer films. 
The authors are grateful to the late Alexander Bune for data in Fig. 2(b), to V. L. Ginzburg for his recommendations concerning this work, and to Evgeny Tsymbal and Alexander Sorokin for helpful suggestions on the manuscript. Work at the Institute of Crystallography of the Russian Academy of
Sciences was supported by the Russian National Science Foundation (RFFI 02-02-06361 and 01-02-16081). Work at the University of Nebraska was supported by the U.S. National Science Foundation (ECS-0070245) and the Nebraska Research Initiative.
${ }^{1}$ W. J. Merz, Phys. Rev. 95, 690 (1954).

${ }^{2}$ E. Fatuzzo and W. J. Merz, Phys. Rev. 116, 61 (1959).

${ }^{3}$ E. Fatuzzo, Phys. Rev. 127, 1999 (1962).

${ }^{4}$ V. M. Fridkin and S. Ducharme, Fiz. Tverd. Tela (St. Petersburg) 43, 1268 (2001) [Phys. Solid State 43, 1320 (2001)].

${ }^{5}$ W. J. Merz, J. Appl. Phys. 27, 938 (1956).

${ }^{6}$ S. Ducharme, V. M. Fridkin, A. V. Bune, S. P. Palto, L. M. Blinov, N. N. Petukhova, and S. G. Yudin, Phys. Rev. Lett. 84, 175 (2000).

${ }^{7}$ K. Kimura and H. Ohigashi, Appl. Phys. Lett. 43, 834 (1983).

${ }^{8}$ V. Ginzburg, J. Phys. (USSR) 10, 107 (1946).

${ }^{9}$ A. F. Devonshire, Adv. Phys. 3, 85 (1954).

${ }^{10}$ T. Furukawa, M. Date, M. Ohuchi, and A. Chiba, J. Appl. Phys. 56, 1481 (1984).

${ }^{11}$ S. Ducharme, S. P. Palto, L. M. Blinov, and V. M. Fridkin, in Proceedings of the Fundamental Physics of Ferroelectrics, edited by R. E. Cohen, (American Institute of Physics, Aspen, CO, 2000), Vol. 535, p. 354.

${ }^{12}$ A. M. Bratkovsky and A. P. Levanyuk, Phys. Rev. Lett. 87, 019701 (2001)

${ }^{13}$ S. Ducharme and V. M. Fridkin, Phys. Rev. Lett. 87, 019702 (2001).

${ }^{14}$ L. D. Landau and I. T. Khalatnikov, Dokl. Akad. Nauk SSSR 96, 469 (1954).

${ }^{15}$ E.-K. Tan, J. Osman, and D. R. Tilley, Phys. Status Solidi B 228, 765 (2001).

${ }^{16}$ D. Ricinschi, C. Harnagea, C. Papusoi, L. Mitoseriu, V. Tura, and M. Okuyama, J. Phys.: Condens. Matter 10, 477 (1998).

${ }^{17}$ S. Sivasubramanian and A. Widom, cond-mat/0106549 (unpublished).

${ }^{18}$ Y. Ishibashi, Jpn. J. Appl. Phys. 31, 2822 (1992).

${ }^{19}$ V. Stephanovich (private communication).

${ }^{20}$ S. Palto, L. Blinov, A. Bune, E. Dubovik, V. Fridkin, N. Petukhova, K. Verkhovskaya, and S. Yudin, Ferroelectr. Lett. Sect. 19, 65 (1995).
${ }^{21}$ L. M. Blinov, V. M. Fridkin, S. P. Palto, A. V. Bune, P. A. Dowben, and S. Ducharme, Usp. Fiz. Nauk 170, 247 (2000) [Phys. Usp. 43, 243 (2000)].

${ }^{22}$ A. V. Bune, V. M. Fridkin, S. Ducharme, L. M. Blinov, S. P. Palto, A. V. Sorokin, S. G. Yudin, and A. Zlatkin, Nature (London) 391, 874 (1998).

${ }^{23}$ Calculations of $\xi=\left(\tau \beta^{2}\right) /(6.3 \gamma)$ were made with the following values for the Landau-Ginzburg coefficients determined from independent measurements (see Ref. 6): $\beta=-1.9 \pm 0.2$ $\times 10^{12} \mathrm{Jm}^{5} / \mathrm{C}^{2}$, and $\gamma=1.9 \pm 0.2 \times 10^{14} \mathrm{Jm}^{9} / \mathrm{C}^{4}$.

${ }^{24}$ A. G. Chynoweth, J. Appl. Phys. 27, 78 (1956).

${ }^{25}$ A. V. Bune, C. Zhu, S. Ducharme, L. M. Blinov, V. M. Fridkin, S. P. Palto, N. N. Petukhova, and S. G. Yudin, J. Appl. Phys. 85, 7869 (1999).

${ }^{26}$ The intrinsic hysteresis loop in Fig. 1(a) would suggest that the curves obtained by this pulsed method must be abrupt; the film would return to its original state at $-P_{\mathrm{S}}$ if $t<t_{0}$ or it would continue to the switched state at $+P_{\mathrm{S}}$ if $t>t_{0}$. The more gradual crossing of $P=0$ in the curves of Fig. 4 is likely due to the inhomogeneous nature of the polycrystalline films. Different crystallites would have different values of the coercive field and damping constant $\xi$ and therefore a range of values for $t_{0}$.

${ }^{27}$ A. Konno, K. Shiga, H. Suzuki, T. Koda, and S. Ikeda, Jpn. J. Appl. Phys. 39, 5676 (2000).

${ }^{28}$ K. Tani, J. Phys. Soc. Jpn. 26, 93 (1969).

${ }^{29}$ R. Blinc and B. Zeks, Soft Modes in Ferroelectrics and Antiferroelectrics (North-Holland, Amsterdam, 1974).

${ }^{30}$ Z. Liu and V. H. Schmidt, Appl. Phys. Lett. 57, 2196 (1990).

${ }^{31}$ H. Dvey-Aharon, T. J. Sluckin, and P. L. Taylor, Phys. Rev. B 21, 3700 (1980).

${ }^{32}$ R. Al-Jishi and P. L. Taylor, Ferroelectrics 73, 343 (1987).

${ }^{33}$ A. Toda, Y. Takahashi, T. Arita, M. Hikosaka, and T. Furukawa, J. Chem. Phys. 114, 6896 (2001).

${ }^{34}$ L. M. Blinov, V. M. Fridkin, S. P. Palto, A. V. Sorokin, and S. G. Yudin, Thin Solid Films 284-285, 474 (1996). 